\title{
Noncontact Measurement for Radius of Curvature of Unpolished Lens
}

\author{
Haifeng Liang \\ College of Photoelectrical Engineering, Xi'an Technological University, Xi'an 710032, China \\ Correspondence should be addressed to Haifeng Liang; hfliang2004@163.com
}

Received 1 July 2014; Accepted 15 September 2014; Published 30 September 2014

Academic Editor: Chenggen Quan

Copyright (c) 2014 Haifeng Liang. This is an open access article distributed under the Creative Commons Attribution License, which permits unrestricted use, distribution, and reproduction in any medium, provided the original work is properly cited.

\begin{abstract}
A noncontact mathematical model to measure radius of curvature (ROC) of an unpolished spherical lens was proposed and also proved by experiments. This model gives ROC as a function of arcs radii and their separation distance, where the radii of the corresponding arcs could be acquired by taking coordinates of points on the arcs when two parallel lines of light project onto a lens surface. Our experiments demonstrated that the measured maximum relative error was $0.027 \%$ for a concave surface with a $38.19 \mathrm{~mm}$ ROC and $0.021 \%$ for a convex surface with a $97.75 \mathrm{~mm}$ ROC, which were all in agreement with those of theory prediction error. The suggested method presented a fast noncontact method for testing ROC of lens during coarse grinding and fine grinding.
\end{abstract}

\section{Introduction}

There are two types of methods by which one can measure the radius of curvature (ROC) for a spherical lens in the optical manufacturing field, namely, contact and noncontact methods [1-5]. Of the contact type, the spherometer and photo-templates gauge are widely used. In order to acquire maximum or minimum sagittal depth when using the spherometer, the probe scans over the surface, which can damage any soft surface but also take long time. In addition, the test results would be easily affected by uncertainty in environmental conditions, such as temperature, mechanical vibration, and different operators. Using the photo-templates gauge, it is easy to damage the soft surfaces of lenses because of similar causes encountered using a spherometer. Furthermore, it is only effective for polished surfaces but ineffective for ground and finely ground surfaces as there are no distinct interference circles.

For the noncontact type, there are also two instruments, namely, the autocollimating microscope [1] and the interferometer [3-5]. In using the former, the radius is equal to the distance between two positions when aligning the center and apex in the proper sequence, respectively [1]. During testing, it is easy to find the apex but very hard to locate the center owing to its uniqueness, thus taking an unduly long time to adjust the optical system. Also, polished surfaces are needed to form an autocollimating image during testing. As is well known, interferometers are rather expensive instruments requiring good environmental conditions to ensure accurate measurements. What is more, these two methods are also only effective for polished surfaces $[2-5]$ because clear image needed to be obtained.

It is necessary for testing the ROC of unpolished surface during coarse grinding and fine grinding to keep appropriate machining allowance for next procedure. As less work has been made to focus this field, a theoretical model of testing ROC was proposed and confirmed through experiments.

The structure of this paper is as follows. Theory model was proposed in Section 2, including its error originates. In Section 3 of experiments and discussions, samples were carried out to prove the validation of this model including analyses of its antienvironment noise. Last, a conclusion, including main contribution, results, and limitation, and future work, was presented.

\section{Theory Model}

A spherical surface of the optical lens could be recognized as part of a complete spherical surface. Hence, measuring the ROC of the lens surface can be obtained by finding the 
corresponding radius of this sphere. The basic theory underpinning the proposed measurement is shown in Figure 1.

Two circles are formed when two parallel planes intersect with sphere. The radii $\left(r_{1}\right.$ and $\left.r_{2}\right)$ of these circles can be obtained in noncontact method. If the distance $(D)$ between these two planes was also priorly known, the ROC of the sphere $(R)$ could be represented as

$$
R=\sqrt{\frac{\left(D^{2}+r_{1}^{2}+r_{2}^{2}\right)^{2}-4 r_{1}^{2} r_{2}^{2}}{4 D^{2}}}
$$

In an actual measurement, only a segment of the circle can be observed (Figure 2(a)) because only the front surface of lens is viewed at any given angle. We represent each of the viewed arcs, as drawn in Figure 2(b), in Cartesian coordinates, and denote the coordinates of the circle center by $\left(x_{0}, y_{0}\right)$ and any point coordinate in the arc by $\left(x_{i}, y_{i}\right)$. According to the circle equation, we have the following formula:

$$
\left(x_{i}-x_{0}\right)^{2}+\left(y_{i}-y_{0}\right)^{2}=r^{2} \text {, }
$$

where $r$ is the radius of the arc. Using just the coordinates of three points on the arc, we can solve this equation to obtain the arc radius. To improve the accuracy, more points can be used to form the set of equations (see (3)) which can be solved using the least squares method. In actual testing, a laser triangulation sensor with a telecentric lens can be used to acquire the relative coordinates of all the points from the two arcs. After acquiring the circle radii and mutual separation between arcs, we can then calculate the ROC using (1). Consider

$$
\begin{gathered}
\left(x_{1}-x_{0}\right)^{2}+\left(y_{1}-y_{0}\right)^{2}=r^{2} \\
\left(x_{2}-x_{0}\right)^{2}+\left(y_{2}-y_{0}\right)^{2}=r^{2} \\
\vdots \\
\left(x_{n}-x_{0}\right)^{2}+\left(y_{n}-y_{0}\right)^{2}=r^{2} .
\end{gathered}
$$

In addition, we see from (1) that the measurement error originates mainly from the mutual distance $(D)$ of the intersecting planes and fitting errors associated with the radii of the arcs. According to the theory of relative error propagation, the relative error $\left(\gamma_{R}\right)$ can be deduced from (1), shown in

$$
\begin{aligned}
\gamma_{R}= & \left|\frac{\partial \ln R}{\partial r_{1}}\right| \Delta r_{1}+\left|\frac{\partial \ln R}{\partial r_{2}}\right| \Delta r_{2}+\left|\frac{\partial \ln R}{\partial D}\right| \Delta D \\
= & \left|\frac{1}{2} \cdot \frac{1}{R^{2} * D^{2}}\left(r_{1} D^{2}-r_{1} r_{2}^{2}+r_{1}^{3}\right)\right| \Delta r_{1} \\
& +\left|\frac{1}{2} \cdot \frac{1}{R^{2} * D^{2}}\left(r_{2} D^{2}-r_{2} r_{1}^{2}+r_{2}^{3}\right)\right| \Delta r_{2} \\
& +\left|\frac{D^{4}-\left(r_{1}^{2}-r_{2}^{2}\right)^{2}}{D \cdot\left(\left(D^{2}+r_{1}^{2}+r_{2}^{2}\right)^{2}-4 r_{1}^{2} r_{2}^{2}\right)}\right| \Delta D .
\end{aligned}
$$

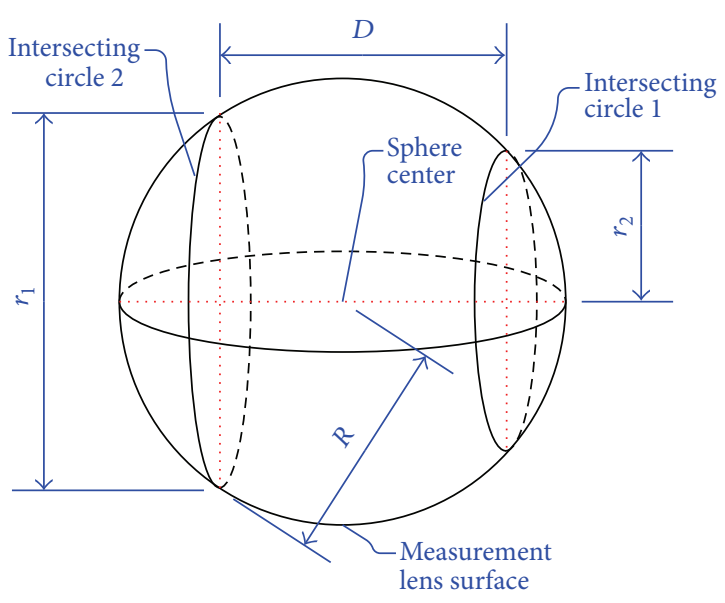

FIGURE 1: Schematic diagram of our method, where $D$ is distance between two parallel planes, $R$ is ROC of the sphere, and $r_{1}$ and $r_{2}$ are the radii of these two intersecting circles, respectively.

In general, $\Delta r_{1}=\Delta r_{2}=\Delta r$; hence (4) is simplified to the following expression:

$$
\begin{aligned}
\gamma_{R}= & \left|\frac{\partial \ln R}{\partial r_{1}}\right| \Delta r_{1}+\left|\frac{\partial \ln R}{\partial r_{2}}\right| \Delta r_{2}+\left|\frac{\partial \ln R}{\partial D}\right| \Delta D \\
= & \frac{1}{2} \cdot \frac{\Delta r}{R^{2} * D^{2}}\left|\left(r_{1}+r_{2}\right)\left(D^{2}-r_{1} r_{2}\right)+r_{1}^{3}+r_{2}^{3}\right| \\
& +\left|\frac{D^{4}-\left(r_{1}^{2}-r_{2}^{2}\right)^{2}}{D \cdot\left(\left(D^{2}+r_{1}^{2}+r_{2}^{2}\right)^{2}-4 r_{1}^{2} r_{2}^{2}\right)}\right| \Delta D,
\end{aligned}
$$

where the error $\Delta D$ is attributed to the calibration error and adjusted to less than $1 \mu \mathrm{m}$. The error $\Delta r$ is ascribed to measurement error of all the coordinates and is deduced from (2). The result is

$$
\begin{aligned}
\Delta r & =\left|\frac{\partial r}{\partial x}\right| \Delta x+\left|\frac{\partial r}{\partial y}\right| \Delta y \\
& =\frac{x}{\sqrt{x^{2}+y^{2}}} \Delta x+\frac{y}{\sqrt{x^{2}+y^{2}}} \Delta y \quad(\text { where } \Delta x=\Delta y) \\
& =\frac{\sqrt{(x+y)^{2}}}{\sqrt{x^{2}+y^{2}}} \Delta x \leq \sqrt{2} \Delta x,
\end{aligned}
$$

where $\Delta x$ and $\Delta y$ include all measurement error during determining the coordinates using the laser triangulation sensor, both having the same level of error.

\section{Experiments and Discussions}

3.1. Theory Discussions. To present a direct idea of the error, we introduce the absolute (repeat) error $(\Delta x)$ of the latest $2 \mathrm{D}$ laser position sensor (LJK series produced by KEYENCE Co. Ltd.) into formula (6) to obtain $\Delta r$; subsequently, $\gamma_{R}$ can be calculated by substituting $\Delta r$ and $\Delta D$ into (5). The absolute 


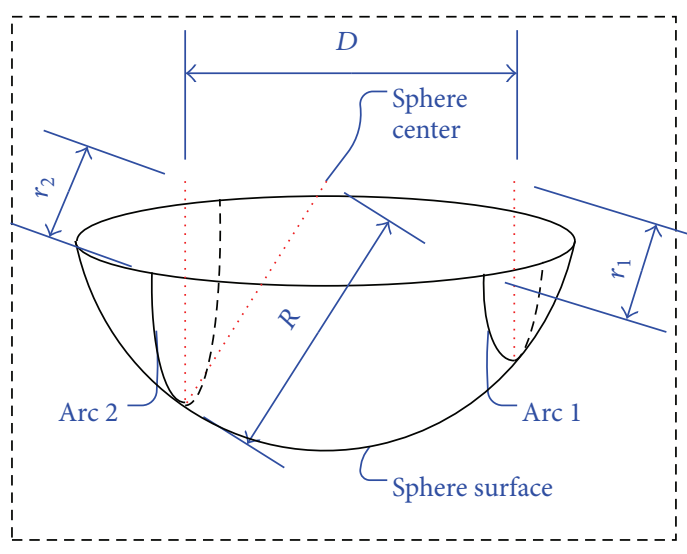

(a)

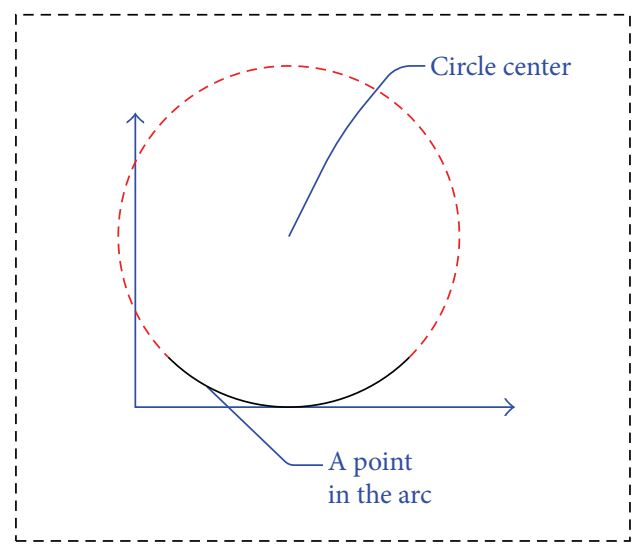

(b)

FIGURE 2: Measurement principle of a concave surface, where $D$ is distance between two parallel planes, $R$ is ROC of the sphere, and $r_{1}$ and $r_{2}$ are the radii of these two intersecting circles, respectively.

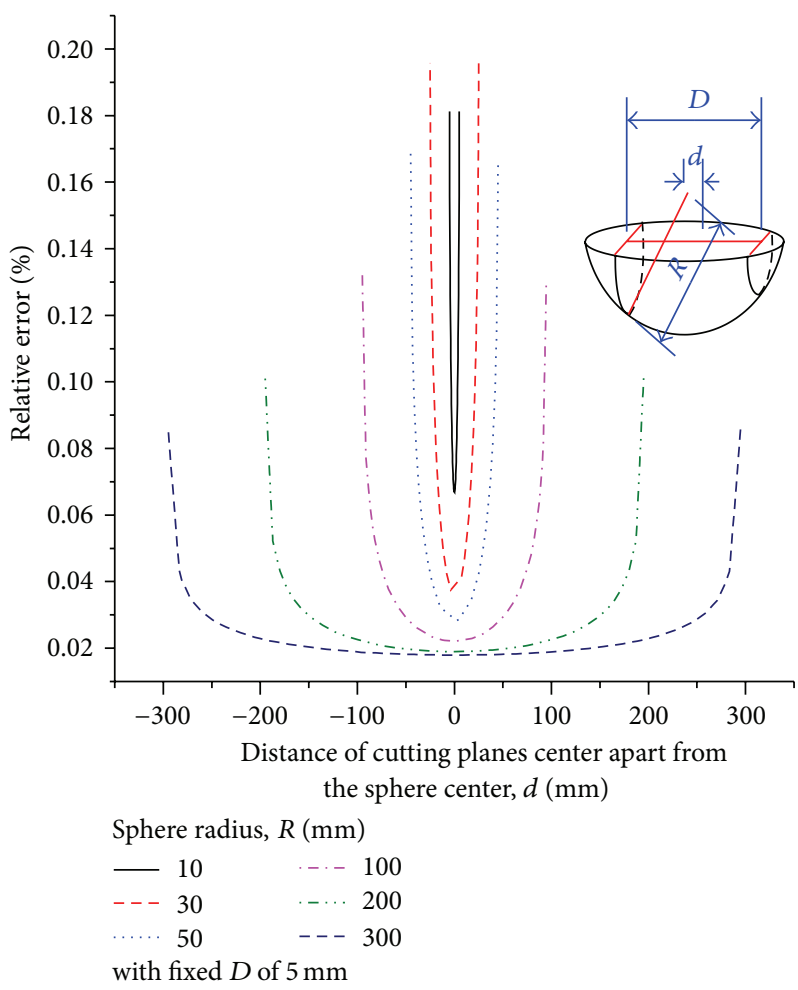

FIGURE 3: Profiles of the relative error for various ROC $(R)$ values with distance $(d)$ of the cutting planes center from the sphere center, where $D$ is distance between two cutting planes.

error for the LJK series sensor is less than $5 \mu \mathrm{m}$, and its measurement range reaches to $26 \mathrm{~mm}$ [6], which is adequate for testing ordinary optical lens.

Curves giving the variation of the relative error with ROC of a lens over the range from $10 \mathrm{~mm}$ to $300 \mathrm{~mm}$ are shown in Figure 3, where the mutual distance $(D)$ of two sphereintersecting planes was set at $5 \mathrm{~mm}$. For any tested ROC, the relative error represents a minimum value when the two sphere-intersecting planes are located in the middle of the lens. We also see that the minimum relative error decreases from $0.065 \%$ to $0.005 \%$ with ROC $(R)$. This is consistent with the empirical rule, namely, high relative error for small ROC and low relative error for large ROC.

Next, the variation of the relative error with intersecting plane mutual distance $(D)$ was also calculated with ROC set at $100 \mathrm{~mm}$ (see Figure 4). With $D$ ranging from $5 \mathrm{~mm}$ to $20 \mathrm{~mm}$, the minimum relative errors were all less than $0.02 \%$, which is similar to that for a commercial spherometer [7]. Relative 


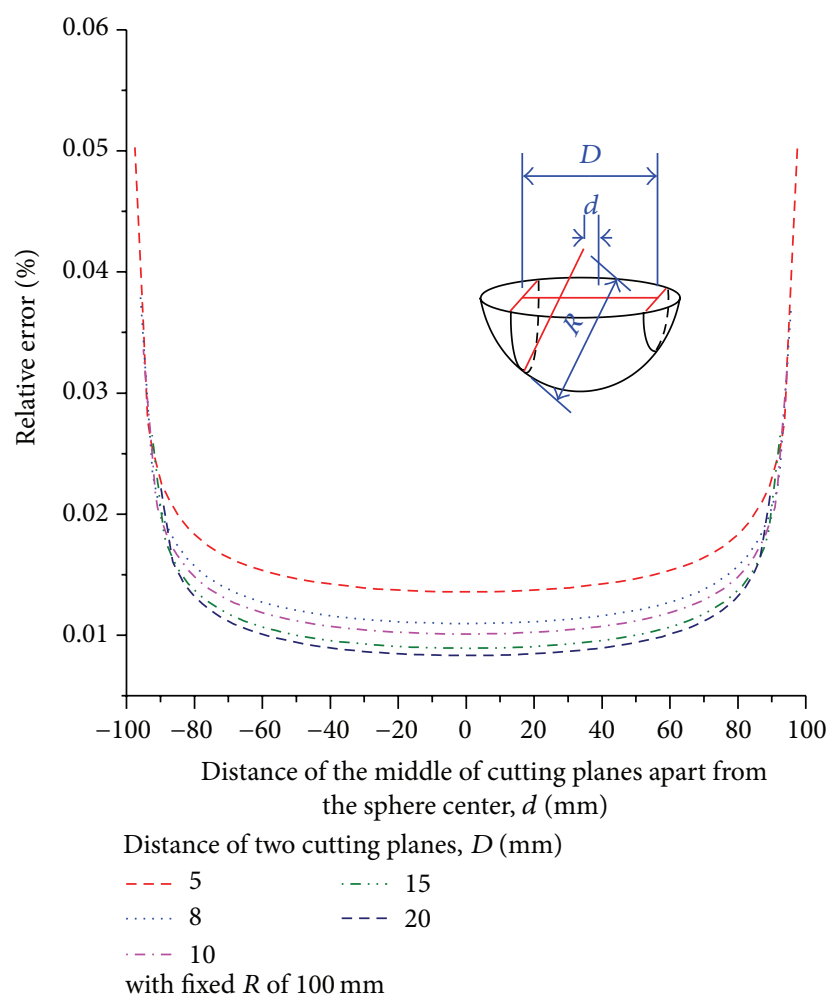

Figure 4: Profiles of the relative error for different mutual distance $(D)$ of cutting planes with the distance $(d)$ of the cutting planes center from the sphere center, where $R$ is ROC of the sphere.

errors also showed a rapid increase as the intersecting planes move away from the center out to the edges. In the intermediate range within the lens, relative errors changed little with relative position and distance between the intersecting planes. This strongly implies that we can randomly set the position of these planes as well as their mutual distance (more than $5 \mathrm{~mm}$ ) during testing.

For most contact or noncontact measurement methods of ROC, such as those mentioned above, the location of the test lens on the holder needs to be precisely aligned using equipment with accurate adjustments $[1-5,7]$. Also, a vibrationless platform is needed for interferometry [3-5], profiler [7], and autocollimating microscopy [1]. These setups are inconvenient for large-scale and high speed measurements.

In our work, the lens only needs to be placed within working distance of the double telecentric lens without accurate aligning. Figure 5 illustrates why position does not affect the error. With the tested lens nearer to the double telecentric lens, the projected arcs do not change because the incident line-structure lights are parallel to each other. Despite the fact that the object distance (between tested lens and double telecentric lens) becomes short, the magnification remains unchanged due to the inherent properties of the double telecentric lens. Thus, the tested lens only requires placing within the working range of the double telecentric lens, which makes the measurement procedure easier and measurement time shorter.

Figure 5(b) shows a diagram illustrating the effect of vibration on the measurement. In such conditions, the tested lens would show inclination with a slight angle to idea position, where two different arc radii to that previous one could be obtained. However, these two new arcs' radii also obey measurement theory, and the difference before and after inclination is that the relative error would increase because these new arcs will not be located symmetrically with respect to the sphere center. Under most conditions, the additional relative error induced by vibration can be ignored because the tilt in angle is very small.

3.2. Experiments. In our experiments, the double telecentric lens with magnification of 0.23 , object distortion of $0.1 \%$, and working distances ranging from 62 to $83 \mathrm{~mm}$ was used to acquire arc images. The projected optical axis was set perpendicular to the lens surface. The angle between projected axis and image axis was fixed at $30^{\circ}$ degree.

A semiconductor laser with output of line-structure light was applied for projecting onto the lens surface. The arcs, spaced $2 \mathrm{~mm}$ apart and labeled (a) through (f) (Figure 6), were imaged by moving the tested lens. In considering the system setup and the calibration parameter, we can then obtain actual coordinates of points on the arcs. Substituting these actual coordinates into (3), we can obtain the radii of each arc using least square fitting with the circle formula. Table 1 lists examples of the results of the procedure. Given the arc radii and separation, the radius of sphere could be calculated according to (1); see column headed "ROC" in Table 1 . The average for the spherical radii was $38.1794 \mathrm{~mm}$, 


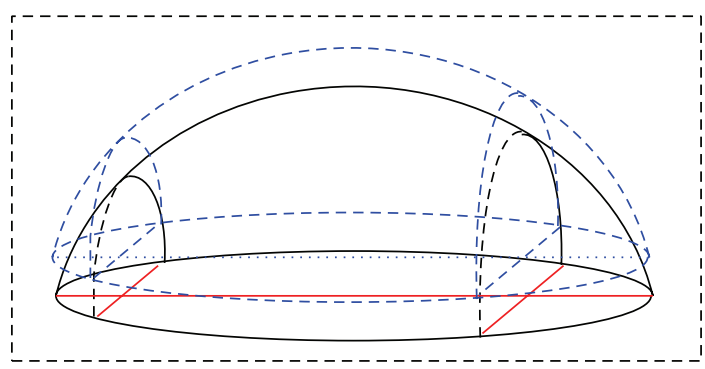

(a)

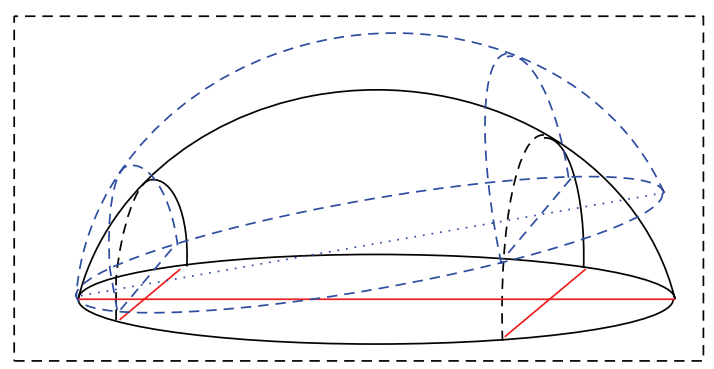

(b)

FIGURE 5: Effect of displacement due to (a) translation and (b) rotation on the ROC measurement during testing, where solid line represents the idea position and dotted line represents the actual position.

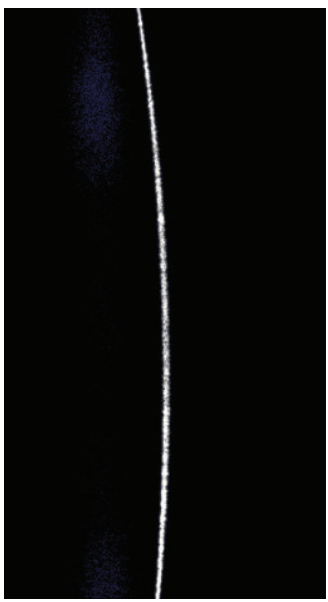

(a)

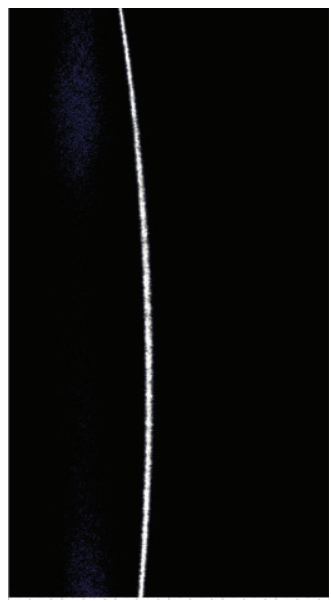

(d)

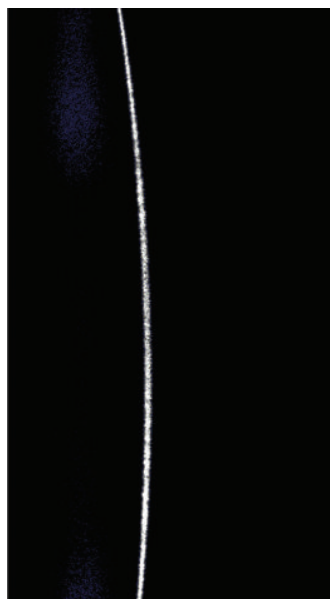

(b)

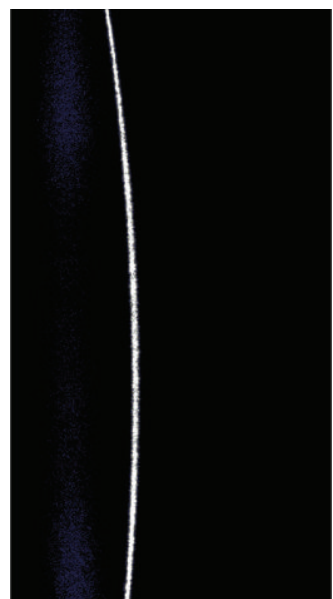

(e)

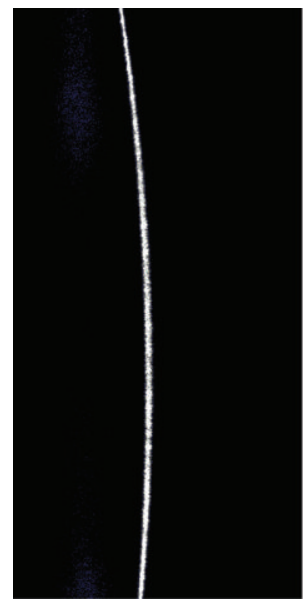

(c)

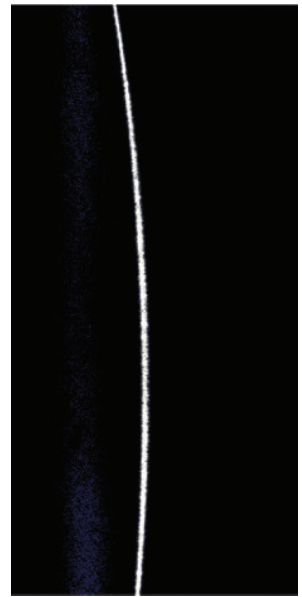

(f)

FIGURE 6: Experimentally obtained image of arcs (part of cutting circles) with $2 \mathrm{~mm}$ moving step of line-structure light.

and the maximum repeatable relative error was only up to $0.0146 \%$. Compared with the nominal value of 38.1900 , the relative error was $0.027 \%$.

Furthermore, a convex surface with a nominal radius of $97.750 \mathrm{~mm}$ was also measured with the same procedure, and the results showed that the average spherical radius was $97.7707 \mathrm{~mm}$, and maximum repeatable relative error was at most only $0.0036 \%$. Compared with the nominal value, the relative error was $0.021 \%$. This error is around one order of magnitude higher than that of interferometer [3-5], which is enough for site testing the ROC of lens during grinding.

\section{Conclusions}

A model for noncontact measurement of the ROC was proposed and confirmed experimentally. This model gives ROC 
TABLE 1: Measurement results for a plane-convex lens.

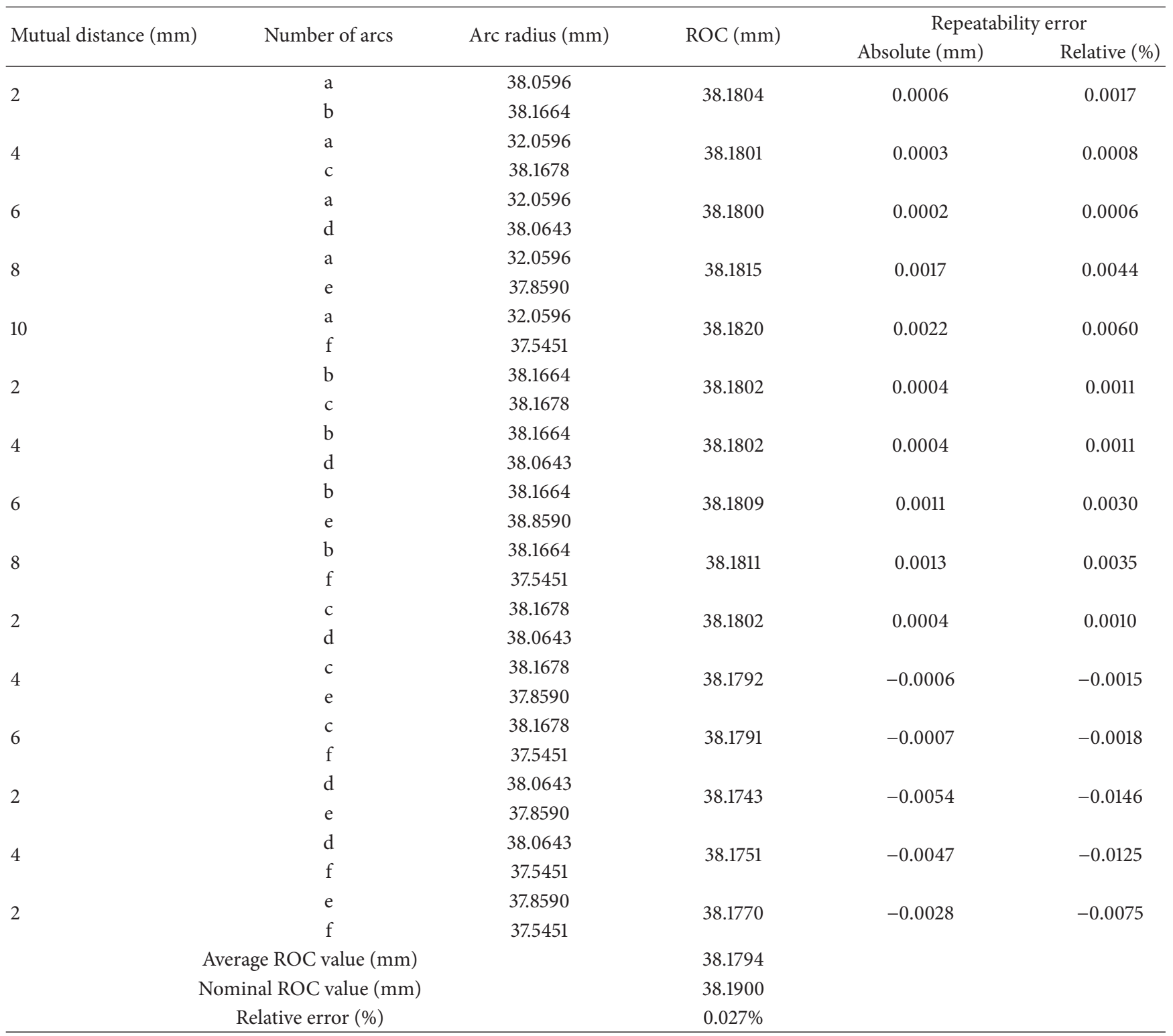

as a function of arcs radii and their separation, where the radii of the corresponding arcs could be acquired by taking coordinates of points on the arcs obtained from two parallel lines of light projecting onto a lens surface. Also, experiments demonstrate that the measured maximum relative error is $0.027 \%$ for a concave surface with a $38.19 \mathrm{~mm}$ ROC and $0.021 \%$ for a convex surface with a $97.75 \mathrm{~mm}$ ROC, which were all in agreement with those of theory prediction error by our model. In addition, the proposed method showed better adaptability against vibration and placement accuracy that results from inherent properties of the measurement principle. Our proposed noncontact method could be applied for site-testing ROC in manufacturing factory. Future work needs to be made on the site experiments for its repeatability through obtaining low noise image of line-structure light and avoiding environment noise.

\section{Conflict of Interests}

The author declares that there is no conflict of interests regarding the publication of this paper.

\section{Acknowledgments}

Thanks are expressed to Dr. Liu Bingcai for experimental help and Dr. Wang Hongjun for discussions on the error propagation.

\section{References}

[1] N. Gardner and A. Davies, "Self-calibration for microrefractive lens measurements," Optical Engineering, vol. 45, no. 3, Article ID 033603, 2006. 
[2] X. Cai, S. Chang, and C. Flueraru, "Compact system for measurement of optical surfaces having a large radius of curvature," Optical Engineering, vol. 45, no. 7, Article ID 073603, 2006.

[3] D. G. Abdelsalam, B. J. Baek, and D. Kim, "Curvature measurement using phase shifting in-line interferometry, single shot offaxis geometry and Zernike's polynomial fitting," Optik, vol. 123, no. 5, pp. 422-427, 2012.

[4] D. G. Abdelsalam, M. S. Shaalan, M. M. Eloker, and D. Kim, "Radius of curvature measurement of spherical smooth surfaces by multiple-beam interferometry in reflection," Optics and Lasers in Engineering, vol. 48, no. 6, pp. 643-649, 2010.

[5] Z. Yang, Z. Gao, Q. Yuan, J. Ye, and M. Li, "Radius of curvature measurement based on wavefront difference method by the point diffraction interferometer," Optics and Lasers in Engineering, vol. 56, pp. 35-40, 2014.

[6] KEYENCE Corporation of America, "LJ-G Series: High-Accuracy 2D Laser Displacement Sensor," http://china.keyence.com/ products/measure/laser2d/ljg/ljg.php.

[7] Trioptics Gmbh, "Super-Spherotronic for high-precision measurements," http://www.trioptics.com/pdfs/Spherometers_Prosp \%20web.pdf. 

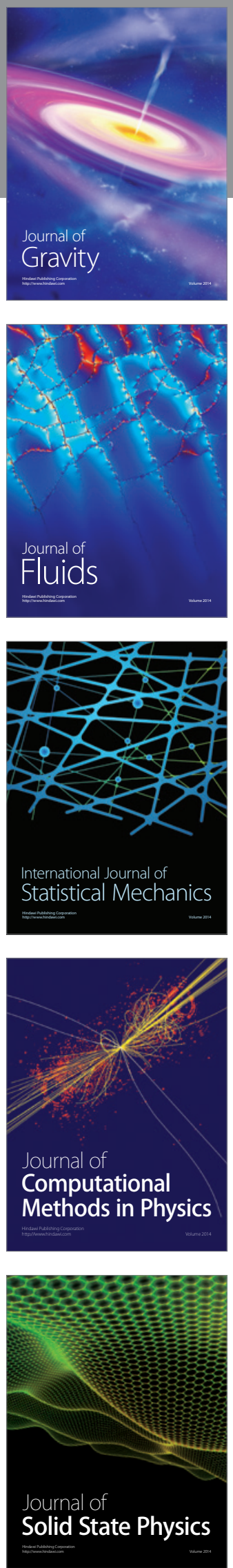

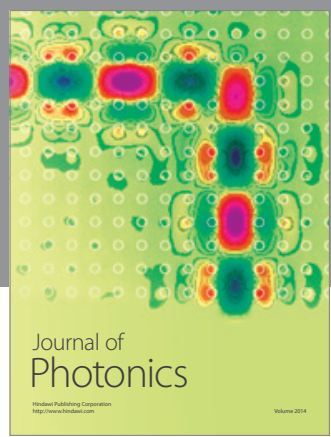

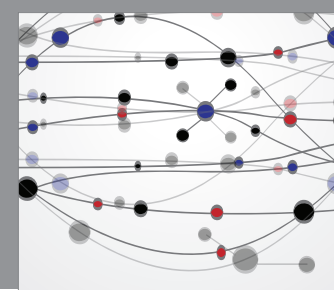

The Scientific World Journal

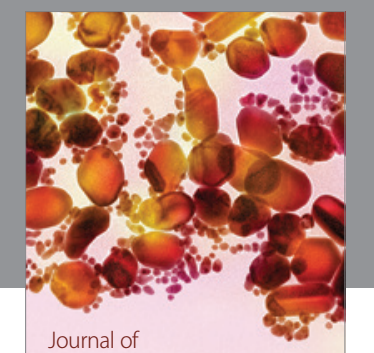

Soft Matter
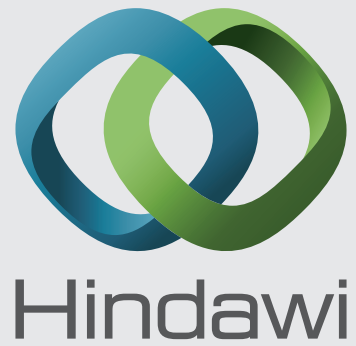

Submit your manuscripts at

http://www.hindawi.com
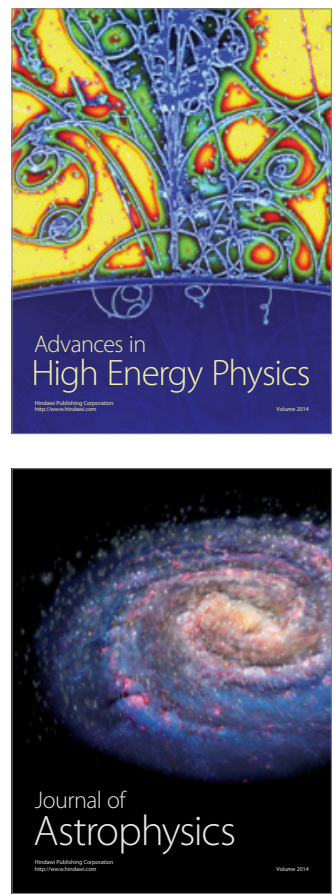
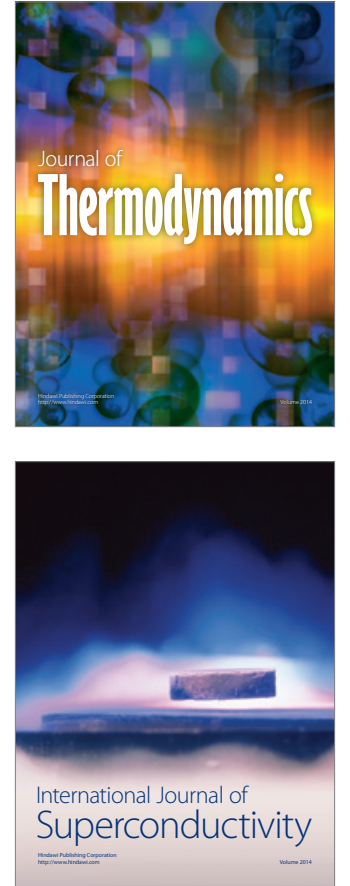
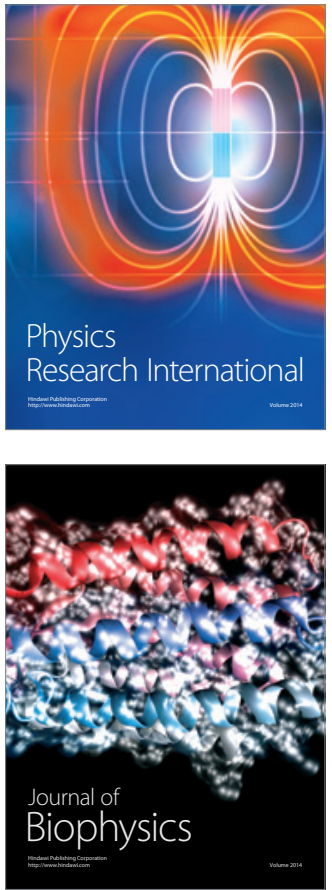
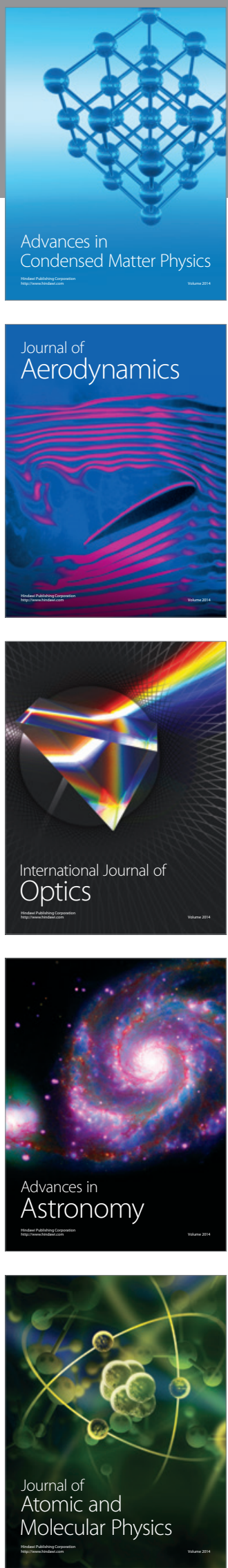